\title{
Hydrogen sensor based on a delay path SAW transducer and a nanostructured catalyst
}

\author{
L. Perez-Cortes ${ }^{1}$, C. Hernandez-Rodriguez ${ }^{1}, \underline{\text { T. Mazingue }}{ }^{1}$ and M. Lomello-Tafin ${ }^{1}$, F. Sarry ${ }^{2}$, G. \\ Lengaine $^{2}$, J.-L. Rousset ${ }^{3}$, F. Morfin ${ }^{3}$ \\ 1 Univ. Savoie Mont Blanc, SYMME, F-74000 Annecy, France, \\ 2 Institut Jean Lamour, UMR 7198, Université de Lorraine-CNRS, Vandœuvre les Nancy, France \\ ${ }^{3}$ Institut de Recherches sur la Catalyse et l'Environnement de Lyon (IRCELYON, CNRS-University of \\ Lyon), 2 Avenue Albert Einstein, F-69626 Villeurbanne Cedex, France \\ thmaz@univ-smb.fr
}

\begin{abstract}
:
Hydrogen sensors are of increasing importance as hydrogen gas is a promising alternative as an energy carrier. Unfortunately, hazardous leaks have to be detected quickly and safely since hydrogen is highly explosive for concentrations in the air within 4 and $75 \%$. This article reports the experimental results of a new scheme of hydrogen detection based on a nanostructured catalyst $\mathrm{PdPt} / \mathrm{Al}_{2} \mathrm{O}_{3}$, a Surface Acoustic Wave (SAW) transducer in a differential configuration, and a signal conditioning circuit. The results show that the scheme of detection presents a linear response, a sensitivity of $5,41 \mathrm{~V} / \%\left(\mathrm{H}_{2}\right)$ and a low drift, in the range of $0.2-2 \%$, with a response time estimated to 10 seconds.
\end{abstract}

Key words: Hydrogen sensor, Surface Acoustic Wave (SAW), palladium and platinum catalyst, signal processing.

\section{Introduction}

Hydrogen $\left(\mathrm{H}_{2}\right)$ has become very attractive as an alternative energy source with zero emission. Unfortunately, this gas is highly explosive when combined with ambient air for concentrations comprised between $4 \%$ and $75 \%$. Sensors with short time responses must be integrated into $\mathrm{H}_{2}-$ based technologies to ensure the safety of use. Most of $\mathrm{H}_{2}$ sensors are based on mature technologies (electrochemical, metal oxide, acoustic and optical detection schemes) that often present a compromise between important expected performances: detection limit, time response, repeatability, cross-sensitivity, reversibility, etc [1]. In this paper, we propose an innovative sensing scheme based on a Surface Acoustic Wave transducer (SAW), and a nanostructured catalyst as sensitive material followed by a signal processing circuit.

\section{Physical principle}

A SAW device is composed of a piezoelectric substrate on which two sets of metallic inputand-output interdigital electrodes (IDT for InterDigital Transducer) are imprinted. They are separated from each other by an area called delay path (DP). An electrical signal applied to the input IDT induces a deformation the of piezoelectric. This deformation propagates as surface acoustic waves along the delay path. These waves are then transformed back into an electrical signal by means of the output IDT, by piezoelectric effect. The attenuation of the transmitted signal is minimal for a value $f_{0}$ of the input signal frequency, called synchronism frequency. The variation of $f_{0}$ with the temperature is described by a parameter called Temperature Coefficient of Frequency (TCF). The output signal presents as well a phase shift $\varphi$ compared to the input signal. $\varphi$ is also directly linked to the temperature [2].

The nanostructured catalyst is composed of an alloy of Palladium and Platinum on Alumina microspheres $\left(\mathrm{PdPt} / \mathrm{Al}_{2} \mathrm{O}_{3}\right)$. This catalyst oxidizes $\mathrm{H}_{2}$ into $\mathrm{H}_{2} \mathrm{O}$ via a highly exothermic reaction $\left(\Delta \mathrm{H}_{25^{\circ} \mathrm{C}}=-241.8 \mathrm{~kJ} \cdot \mathrm{mol}^{-1}\right)$ at room temperature. The heat produced during the reaction leads to a temperature increment of the catalyst, with a first-order response. We have previously shown that the temperature increase is directly linked to the concentration of $\mathrm{H}_{2}$ [2].

Once the catalyst is deposited on the DP of the SAW, it is possible to observe a variation of the phase shift compared to a SAW reference, under $\mathrm{H}_{2}$ exposure, due to the temperature increment. A signal conditioning system (composed of an impedance coupler, a phase detector and a 
microcontroller) performs numerical operations to minimize undesired noise and executes calculations (temporal derivative) to return a voltage related to the $\mathrm{H}_{2}$ concentration.

\section{Results}

The sensor is exposed to alternating sequences of $30 \mathrm{~s}$ of dry synthetic air and a given concentration of $\mathrm{H}_{2}$ (from 0,2 to $2 \%$ ). The experiences are carried out at a flow rate of 500 $\mathrm{mL} . \mathrm{min}^{-1}$ and at room temperature.

As shown in fig.1, the first-order thermal response of the catalyst is detected by the SAW transducer (blue curve) by means of phase shift compared to the SAW reference (differential measurement). The signal is not stabilized after an exposure of $30 \mathrm{~s}$ to different concentrations of $\mathrm{H}_{2}$, and it is therefore impossible to associate the temperature increase measured with the $\mathrm{H}_{2}$ concentration. In order to solve this problem, a time derivative is applied aiming to detect the breaking point of the slope observed when $\mathrm{H}_{2}$ is injected into the measurement chamber. The derivative returns a peak to each slope breaking point (green curve). The amplitude of the peak is directly linked to $\mathrm{H}_{2}$ concentration, as explained in reference [2]. The sensor output is then defined by the time derivative of the signal coming from the phase detector (green curve). The time response $\tau$ is determined by the time elapsed from the injection of $\mathrm{H}_{2}$ in the measurement chamber (slope on the blue curve) and the apparition of the peak (green curve). It is estimated to $10 \mathrm{~s}$ approximately.

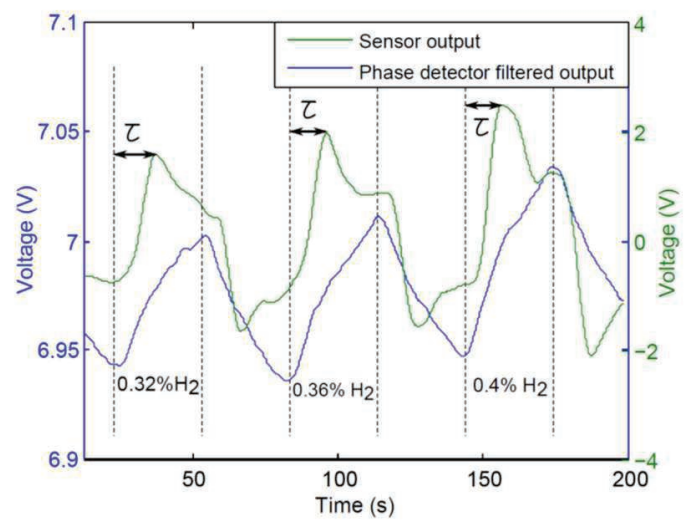

Fig. 1. Signal at the output of the phase detector (in blue) and signal at the output of the sensor (in green) for different $\mathrm{H}_{2}$ concentrations.

The sensor has been exposed at least 3 times to $\mathrm{H}_{2}$ concentrations in dry air ranging from 0.2 to $2 \%$. Figure 2 plots the height of the peak of the derivative, considered as the sensor output, vs the concentration, including error bars. We can observe that the sensor response is linear for $\mathrm{H}_{2}$ concentrations within the explored range, with a correlation coefficient $\mathrm{R}^{2}=0.9963$. The repeatability has been estimated more accurately by 10 repetitive measurements for $\mathrm{H}_{2}$ concentration at 0.32 and $1 \%$. In the worst case, the deviation reached $4.4 \%$. At last, the sensitivity $S$ is estimated by the slope of the straight line fitted by the linear regression: $S=$ $5.41 \mathrm{~V} / \%$.

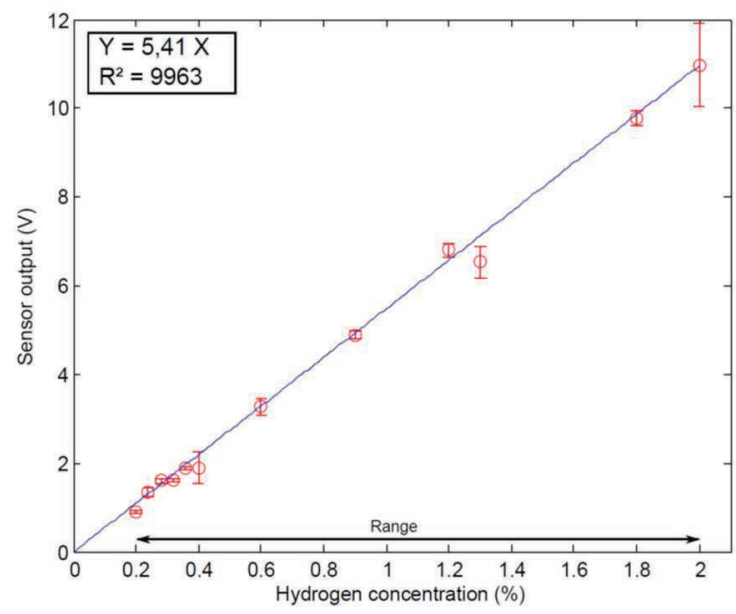

Fig.2.Response of the sensor for different concentrations of $\mathrm{H}_{2}$ in dry synthetic air

\section{Conclusions}

The feasibility of $\mathrm{H}_{2}$ concentration measurement by measuring the heat generated by an exothermic oxidation on a nanostructured catalyst with a SAW transducer has been demonstrated. The prototype we developed presents promising performances in terms of time response (< $10 \mathrm{~s})$, repeatability and reversibility at room temperature, for a sensitivity of $5.41 \mathrm{~V} / \%$. However, this work has to be completed by an exhaustive metrology work (influence of external parameters such as relative humidity, temperature, cross-sensitivity to other gases, signal drift, etc...). This new technology presents an attractive alternative for measuring hydrogen concentrations passively. Wireless interrogation can indeed be considered in a future work, with a differential device connected to antennas for an autonomous transducer configuration.

\section{References}

[1] T. Hübert, L. Boon-Brett, G. Black, U. Banach, Hydrogen sensors - a review, Sensors and Actuators B 157, 329-352 (2011); doi.org/10.1016/j.snb.2011.04.070

[2] T. Mazingue, M. Lomello-Tafin, M. Passard, C. Hernandez-Rodriguez, L. Goujon, J.-L. Rousset, F. Morfin and J.-F. Laithier, Catalytic and thermal characterisations of nanosized $\mathrm{PdPt} / \mathrm{Al}_{2} \mathrm{O}_{3}$ for hydrogen detection Journal of Sensors and Sensor Systems., 3, 273-280 (2014); doi:10.5194/jsss-3-273-2014 